\title{
Slow proton production in deep-inelastic neutrino scattering on deuterium
}

\author{
G.D. Bosveld, A.E.L. Dieperink \\ Kernfysisch Versneller Instituut, NL-9747 AA Groningen, The Netherlands \\ A.G. Tenner \\ NIKHEF-H, P.O.Box 41882, NL-1009 DB Amsterdam, The Netherlands
}

\begin{abstract}
The cross section for semi-inclusive deep inelastic charged current neutrino scattering on hydrogen and deuterium in which a slow proton is observed in coincidence with the muon is computed as a function of Bjorken $x$ and light-cone momentum of the detected proton. In the Impulse Approximation contributions from hadronization and (in case of the deuteron) the emission of a spectator nucleon are considered. In addition the probability for rescattering is computed. The results are compared to a recent analysis of the data from the WA25 (BEBC) experiment at CERN.
\end{abstract}

25.30.Pt,13.85.Hi

Typeset using REVTEX 


\section{INTRODUCTION}

Inclusive deep inelastic lepton scattering on nucleons is a well established tool for the investigation of the quark-parton model. In the Bjorken limit the cross section is proportional to a structure function which depends on only one scaling variable $x$, that can be interpreted as the light-cone momentum fraction of the hit quark. Deep inelastic scattering on nuclear targets showed [1] that the $x$ distribution of quarks in nucleons bound in a nucleus differs from the one for a free nucleon (EMC effect). While part of this effect can be explained in terms of nuclear binding and fermi motion [2, [3] there is evidence that additional possibly non-nucleonic effects are required to obtain agreement with the data. However, since in inclusive experiments one averages over all, strongly and weakly bound nucleons, it is not possible to draw definite conclusions about the nature of these effects.

More detailed information on the deep inelastic scattering process can be obtained from more exclusive experiments. Here we concentrate on semi-inclusive deep inelastic scattering (SIDIS) on the deuteron in which at least one slow (i.e. low energy) proton is detected in coincidence with the scattered lepton. There are several reasons why this process is of interest both on a free nucleon and on nuclear targets.

On a free nucleon slow protons originate from hadronization of the spectator quarks in the struck nucleon (so-called target fragmentation). In a previous study [4, (4] SIDIS on a free proton was considered, assuming factorization of the cross section into a structure function $F(x)$ and a spectator quark (debris) fragmentation function $D(z)$, where $z$ is the light-cone momentum fraction carried by the observed proton. It was found that the ratio $P(x, z)$ of the SIDIS cross section $\sigma(x, z)$ and the inclusive cross section $\sigma(x)$ has a strong bias for small $x$ values (in agreement with experiment). Qualitatively this behavior can be explained by kinematics: a proton at rest in the target rest system can only be produced at $x=0$; on the other hand the dependence on $z$ and some details of $x$ dependence are sensitive to the shape of the fragmentation function.

On nuclear targets there are several mechanisms that can give rise to slow protons. 
First there is the above mentioned debris fragmentation of the quarks in the struck bound nucleon, giving rise to creation of new particles and possible change of momenta of spectator nucleons. From a comparison with the free nucleon one obtains information on the effects of the nuclear medium on the hadronization process.

Another category of slow protons in deep inelastic scattering on nuclei are spectator nucleons which are emitted from nucleon-nucleon correlations present in target ground state. In the special case of pure two-body correlations the l.c. momentum fraction $z$ of an observed spectator proton directly determines the l.c. momentum of the nucleon being struck by the lepton, $y \approx 2-z$. In this case one deals with the tagged EMC effect (originally proposed by Frankfurt and Strikman [6]. This is of interest since it might help to determine whether the convolution approach to the EMC effect is appropriate [3, ,7], or non-nucleonic degrees of freedom are required [8,9].

Whereas in scattering on a free nucleon the direct hadronization process is the only one possible, in nuclei both the direct and spectator processes occur and it is of interest to investigate their characteristic behavior. In this paper we concentrate on the simplest possible nucleus, the deuteron. We compute the double differential cross section $\sigma(x, z)$ for the production of slow protons with l.c. momentum fraction $z$. For the experimental data we use the result of a recent new analysis of the WA25 collaboration. In the latter a (partial) decomposition of the (anti-)neutrino SIDIS cross section is achieved in terms of spectator, direct and rescattering events. In particular the spectator contribution can be uniquely identified by sampling the slow protons in the backward hemisphere. Thus it allows one to compare the various processes separately with experimental results.

Another interesting feature of the deuteron target is that it provides information on the fragmentation of the neutron into a slow proton in (anti-)neutrino induced reactions. In a pure valence quark approach the cross section for $\nu+n \rightarrow \mu+p+X$ would vanish. In practice one must also take into account sea-quark contributions (important at small $x$ ). The latter can be treated in two ways, namely as a 4-quark fragmentation or as a bag of valence quarks surrounded by a pion cloud, which is struck by the lepton. While these models give 
similar results for inclusive processes there are interesting differences for exclusive reactions. For example, in the pion model one can produce slow protons in $\nu+n$ in two ways: via $n \rightarrow p+\pi^{-}$, and via $n \rightarrow \Delta^{0}+\pi^{0}$ with subsequent decay of the isobar into $p+\pi^{-}$, whereas in the naive leading order 4-quark fragmentation only the latter process contributes to the production of slow protons

Finally, in the present study we discuss possible effects of final state interactions. After hadronization of the quarks in the hit nucleon (determined by the formation time) the hadrons which are produced in forward direction can in general interact with the spectator nucleons and thus lead to a possible modification of their momentum distribution and charge and the creation of new particles. The possibility to study the dependence of rescattering on the formation (hadronization) time is of interest since it plays an essential role in the interpretation of heavy ion reactions.

The paper is organized as follows. In section 2 we summarize the SIDIS cross section on a free proton. The hadronization is described in terms of fragmentation functions for diquarks and four-quarks. In section 3 we discuss the direct and spectator contribution for the deuteron assuming plane wave impulse approximation and compare with experiment. The effect of rescattering on the spectator momentum distribution is estimated in the eikonal approximation and the role of the formation time is discussed. Section 4 contains concluding remarks.

\section{SLOW PROTON PRODUCTION ON A NUCLEON}

\section{A. Introduction}

In the Bjorken limit inclusive deep inelastic scattering (DIS) on a nucleon is characterized (in leading order) by one scaling variable $x=Q^{2} / 2 p \cdot q$, where $Q^{2}=-q^{2}$ is the square of the four-momentum transfer and $p$ the nucleon momentum. In the parton model a DIS event at a specific $x$ corresponds to the knock-out of a quark carrying a fraction $x=-q^{-} / p^{-}$of 
the nucleon light-cone momentum (where $q^{ \pm}=q^{0} \pm q^{3}$ and the momentum transfer $\vec{q}$ is in the direction of $z$ ). In case of neutrinos the cross section can be expressed in terms of three structure functions $F_{i}(x),(i=1,2,3)$ which only depend upon the quark momentum distributions.

The physical picture immediately after the (leptonic) scattering process is that of one quark moving with a high momentum with respect to the debris of the target (the quark cluster), cf. Fig. 1. Ultimately, the quark and the cluster will interact via the strong color field, in which quark-antiquark pairs are produced, yielding color neutral objects in the final state.

In semi-inclusive deep inelastic scattering (SIDIS) one observes one or more particles in coincidence with the scattered lepton. For our purposes it is convenient to distinguish between two limiting processes, namely the production of mainly fast particles originating from the fragmentation of the struck leading quark, and the production of mainly slow hadrons coming from fragmentation of the debris. As to the leading quark fragmentation one can show [10] that in leading order (in $1 / Q$ ) the cross section can be factorized into a structure function $F(x)$ which describes the DIS on a quark and a probability for hadronization. The latter can be parametrized by a fragmentation function $D_{\{q\}}^{\mathrm{x}}\left(z_{f}, p_{\perp}\right)$ with the property that $D_{\{q\}}^{\mathrm{x}}\left(z_{f}, p_{\perp}\right) d z_{f} d p_{\perp}$ is the probability that a 'jet' with quark-content $\{q\}$ yields a fragment $\mathrm{x}$ carrying a fraction $z_{f}=p_{\mathrm{x}}^{+} / q^{+}$of the initial quark light-cone momentum and transverse momentum $p_{\perp}$.

The fragmentation process of the quarks left behind (debris or target fragmentation) is more complicated. In this case one cannot use perturbative QCD arguments to prove factorization even in leading order [10]. Still in practice one usually also assumes that the cross section factorizes. The appropriate variable for target fragmentation is $z_{s}=p_{\mathrm{x}}^{-} /\left(p^{-}+\right.$ $q^{-}$) which is related to the observable fraction of the target momentum: $z=p_{\mathrm{x}}^{-} / p^{-}=$ $(1-x) z_{s}$ 


\section{B. Semi inclusive cross section}

The angle integrated semi-inclusive cross section for deep inelastic (anti-)neutrino scattering on a nucleon with the observation of a hadron $\mathrm{x}$ with l.c. momentum fraction $z_{s}$ and transverse momentum and $p_{\perp}$ can be written as

$$
\frac{d^{3} \sigma^{\bar{\nu}, \nu}}{d x d z_{s} d p_{\perp}}=\frac{G^{2} m E}{\pi}\left[\frac{x}{3} \mathcal{F}_{1}+\frac{1}{2} \mathcal{F}_{2} \pm \frac{x}{3} \mathcal{F}_{3}\right]
$$

with $G$ the Fermi coupling constant and $E$ the energy of the (anti-)neutrino. From now on we will only consider target fragmentation. In the factorized approximation the semi-inclusive proton structure functions for neutrino scattering $\mathcal{F}_{i}^{\nu p}$ can be expressed as

$$
\begin{aligned}
& \mathcal{F}_{2}^{\nu p}\left(x, z_{s}, p_{\perp}\right)=2 x\left[d(x) D_{\{p / d\}}^{\mathrm{x}}\left(z_{s}, p_{\perp}\right)+\bar{u}(x) D_{\{p / \bar{u}\}}^{\mathrm{x}}\left(z_{s}, p_{\perp}\right)\right], \\
& \mathcal{F}_{3}^{\nu p}\left(x, z_{s}, p_{\perp}\right)=2\left[d(x) D_{\{p / d\}}^{\mathrm{x}}\left(z_{s}, p_{\perp}\right)-\bar{u}(x) D_{\{p / \bar{u}\}}^{\mathrm{x}}\left(z_{s}, p_{\perp}\right)\right],
\end{aligned}
$$

and $\mathcal{F}_{1}=\mathcal{F}_{2} / 2 x$. Here $d(x)$ and $\bar{u}(x)$ are the down-and anti-up quark momentum distributions in the nucleon, respectively; $p / d$ and $p / \bar{u}$ denote the cluster resulting after the knockout of a down and anti-up quark from a proton, respectively. The structure functions for anti-neutrinos are obtained from those of the neutrinos by replacing $d \leftrightarrow \bar{d}$ and $u \leftrightarrow \bar{u}$.

Since the neutrino can interact with both valence and sea quarks, the number of quarks in the debris (cluster) varies. When a valence quark is hit, a diquark cluster remains, while

the knockout of a sea quark (which is expected to be important at small $x$ values) results in (at least) a four quark cluster. The fragmentation functions for these processes are expected to have a different dependence on $z$ [1]. Hence it is convenient to decompose the semiinclusive cross sections for neutrino proton scattering into a valence and a sea contribution, $\sigma^{\nu p}=\sigma_{\text {val }}^{\nu p}+\sigma_{\text {sea }}^{\nu p}$, with

$$
\frac{d^{3} \sigma_{\mathrm{val}}^{\nu}}{d x d z d p_{\perp}}=\frac{G^{2} m E}{\pi} \frac{2 x}{1-x} d_{v}(x) D_{\{u u\}}^{p}\left(\frac{z}{1-x}, p_{\perp}\right),
$$

and

$$
\frac{d^{3} \sigma_{\mathrm{sea}}^{\nu}}{d x d z d p_{\perp}}=\frac{G^{2} m E}{\pi} \frac{2 x}{1-x}\left[d_{s}(x) D_{\left\{p / d_{s}\right\}}^{p}\left(\frac{z}{1-x}, p_{\perp}\right)+\frac{1}{3} \bar{u}_{s}(x) D_{\left\{p / \bar{u}_{s}\right\}}^{p}\left(\frac{z}{1-x}, p_{\perp}\right)\right] .
$$


Here the subscript $\left\{p / q_{s}\right\}$ denotes the four-quark system $\left\{u u d \bar{q}_{s}\right\}$ and we have neglected strange quarks. For slow fragments (i.e. $z \approx 1$ ) the diquark fragmentation function $D_{\{q q\}}^{\mathrm{x}}$ can be approximated by a splitting function [12], multiplied by combinatorial flavor/isospin factors $\gamma_{\{q q\}}^{\mathrm{x}}$ and a transverse momentum distribution

$$
D_{\{q q\}}^{\mathrm{x}}\left(z_{s}, p_{\perp}\right)=\lambda_{q q} \gamma_{\{q q\}}^{\mathrm{x}}\left(z_{s}-z_{\min }\right) \theta\left(z_{s}-z_{\min }\right) f\left(1-z_{s}\right) \rho\left(p_{\perp}\right),
$$

where $z_{\min }$ (corresponding to a proton at rest in the hadronic center-of-mass frame) is given by $z_{\min }=\sqrt{m^{2}+p_{\perp}^{2}} / W$ with $W$ the invariant mass of the final system, $W^{2}=m^{2}+Q^{2}(1 / x-$ 1) [13]. For the transverse momentum distribution we assume the form

$$
\rho\left(p_{\perp}\right)=\frac{4 p_{\perp}}{\left\langle p_{\perp}\right\rangle^{2}} e^{-2 p_{\perp} /\left\langle p_{\perp}\right\rangle},
$$

with $\left\langle p_{\perp}^{2}\right\rangle \approx 300(\mathrm{MeV} / c)^{2}$. For the splitting function $f$ we use the counting rule estimate $f(\xi)=\xi^{n} \quad($ with $n=1)$ [14,15]. The factors $\gamma_{\{q q\}}^{\mathrm{x}}$ are given in Table [. The overall normalization $\lambda_{q q}$ can be obtained by requiring that the integral of $D$ over the appropriate $z$ range is normalized to unity,

$$
\sum_{\mathrm{x}} \int_{z_{\min }}^{1} d z_{s} D_{q q}^{\mathrm{x}}\left(z_{s}\right)=1
$$

The fragmentation of the four-quark system (resulting from the interaction with a seaquark) has not been dealt with in any detail in the past. Ishii et al. 16 assume a unit probability for fragmentation into a nucleon. In the present study we need in addition to the $z$ dependence a prescription for the relative branchings into $p, n$ and the various isobars. Since for the later application to $A=2$ it is convenient to use a simple form; in the present paper we use the parametrization [13]

$$
D_{\{4 q\}}^{\mathrm{x}}\left(z_{s}\right)=\lambda_{4 q} \gamma_{\{4 q\}}^{\mathrm{x}} \theta\left(z_{s}\right) \theta\left(1-z_{s}\right)
$$

We will consider two possible choices for $\gamma_{\{4 q\}}^{\mathrm{x}}$ :

(i) The most naive approach [16] is to assume that the produced baryon has the same valence quark structure as the hit nucleon (i.e. $p \rightarrow p, \Delta^{+}$and $n \rightarrow n, \Delta^{0}$ ). The resulting $\gamma_{\{4 q\}}^{\mathrm{x}}$ are listed in Table [1]. 
(ii) Alternatively we can simulate the sea quarks by a meson cloud model. Such a model has recently been used to describe the sea-contribution in SIDIS; it is a very effective mechanism for the production of slow nucleons [13, 5, 17]. In this case the coincidence cross section is given by 17

$$
\frac{d^{3} \sigma_{\mathrm{sea}}^{\nu}}{d x d z d t}=\frac{G^{2} s}{2 \pi}(1-z) \frac{g_{\pi N N}^{2}}{(4 \pi)^{2}} \frac{t}{\left(t+m_{\pi}^{2}\right)^{2}} F_{2}^{\nu \pi}\left(\frac{x}{1-z}\right)\left|F_{\pi N N}(t)\right|^{2}
$$

where $t=-\left(p_{\mathrm{x}}-p\right)^{2}, g_{\pi N N}$ is the pion nucleon coupling constant and $F_{\pi N N}(t)$ the corresponding form factor. (The corresponding expression for the production of a Delta is given in Ref. [17].)

In practice it is convenient to use the factorized form of Eqs. (5) and (9), retaining only the branching ratios $\gamma_{\{4 q\}}^{\mathrm{x}}$ that result from the meson cloud model. These involve the different isospin couplings at the $\pi N N$ and $\pi N \Delta$ vertices (which determine the relative magnitudes of $g_{\pi N N}$ and $g_{\pi N \Delta}$ ), the different couplings of the probe with the neutral and charged mesons and a parameter $\kappa$ which determines the relative ratio of nucleon and isobar production. The latter is taken equal to one [17]. The resulting values for values for $\gamma_{\{4 q\}}^{\mathrm{x}}$ are listed in Table [II]. Analogously to Eq. (8) the $\lambda_{4 q}$ follows from the normalization of the four-quark fragmentation function is such that

$$
\sum_{\mathrm{x}} \int_{z_{\min }}^{1} d z_{s} D_{\{4 q\}}^{\mathrm{x}}\left(z_{s}\right)=1
$$

One noticeable difference between the above two prescriptions is that in a $\nu+n$ interaction a proton can be produced in approach (ii) but not in (i).

The probability for finding a slow proton in a momentum bin $\Delta p$ is obtained by integrating the triple differential cross section over the corresponding $\left(p_{\perp}, z\right)$ domain and dividing by the inclusive cross section

$$
P(x, \Delta p)=\int_{\Delta p} d z d p_{\perp} \sigma\left(x, z, p_{\perp}\right) / \sigma(x)
$$

Note that, apart from the direct production of protons, there is also a significant contribution from decay of nucleon isobars. This is taken into account by assuming that the resonances decay isotropically in their rest frame 17,5]. 
In Fig. 2 we show the ratio $P(x, \Delta p)$ of the calculated SIDIS cross section and the inclusive cross section as a function of $x$ for (anti-)neutrino scattering on the free proton compared with the data of Guy et al. [18]. Since in that paper only a relative ('renormalized') cross section was given we normalized the published data to our calculated cross section in the low bin for $\nu+p$.

For comparison in Fig. 3 the neutron the experimental cross section as extracted from the deuterium experiment (as discussed in the next section) is shown. The following points are worth noting:

(i) Whereas the proton data have an arbitrary normalization, it should be emphasized that the neutron data are absolute.

(ii) Qualitatively the fall off of $P(x, \Delta p)$ with $x$ can be ascribed to kinematics: slow protons correspond to $z \approx 1$ while $z+x<1$. Only the details of the dependence on $x$ are sensitive to the precise form of the fragmentation function. The presence of an experimental tail both in the $\nu+p$ and $\nu+n$ data which extends beyond the kinematical limit, $x=1-z$, is puzzling and cannot be explained. The larger tail in the proton data may be due to the absence of an unsmearing of $x$ in the analysis. (In ref. [13] this taken into account by an (ad hoc) smearing of the $x$ distribution.)

(iii) The ratio of $P(x, \Delta p)$ for the low and high bin, which depends on the form of the fragmentation function near $z=1$, is somewhat underestimated; the sea contribution dominates at small $x$ values and is more important for the low bin than in the high bin.

(iv) Experimentally, $P(x, \Delta p)$ for $\nu$ and $\bar{\nu}$ scattering on a neutron are quite different, and almost equal for a proton target. For the $\bar{\nu}+n$ process the valence contribution almost vanishes, and the cross section is strongly affected by sea-quark effects; the sea contribution is larger when using the pionic model than naive fragmentation.

(v) Comparing the different choices for the 4q fragmentation function one sees that in $\bar{\nu}+p$ the naive 4q fragmentation yields a larger cross section than the pion model while in $\nu+n$ one has the opposite situation. This is a consequence of the presence of an important charged meson exchange contribution in the pion cloud approach. 
(vi) The unfavoured fragmentation process $\bar{\nu}+n \rightarrow \mu^{+}+p+X$ which does not have a direct contribution proceeds only via an intermediate $\Delta^{0}$ after scattering on the sea-quarks in the pion cloud approach. The experimental cross section does not show such a dramatic effect.

\section{THE DEUTERON}

\section{A. Classification of events}

In a nuclear medium there are several processes that can give rise to slow protons. First in analogy with the DIS on a free nucleon target slow protons can originate from fragmentation of the spectator quarks (debris fragmentation) in the struck nucleon with the other nucleons acting as spectators. This process will be referred to as the direct process. A new effect, which only occurs for $A>1$, is the deep inelastic scattering off a bound nucleon and the observation of a spectator proton (called the spectator process). Since in a mean field description the $A-1$ nucleus is bound, this process provides evidence for the presence of correlations in the ground state (for $A>2$ ). The important point is that in the independent pair approximation neglecting the centre-of-mass motion of the pair and final state interactions one has a direct relation between the l.c. momentum fractions of the detected $(z)$ and struck $(y)$ nucleons $y+z=2$.

In the following we will compute the SIDIS cross section on the deuteron with a slow proton present assuming that the above processes contribute incoherently. An additional process that will be considered separately is the possible reinteraction (rescattering) of the hadrons produced in the fragmentation with the spectator nucleon. The latter process is of interest since it depends on the hadronization in the nuclear medium, which may lead to a modification of the momentum and possibly the charge of the observed spectator nucleon.

The data to be used as a comparison were obtained from an analysis of deep inelastic (anti-)neutrino scattering experiment on the deuteron in a bubble chamber in which all (charged) particles were detected [19]. The experimental data allow for a separation into 
'even' and 'odd' events, corresponding to an even or odd number of charged hadrons in the final state, respectively. From charge conservation it follows that odd events correspond to the direct process on the neutron, where the spectator proton has insufficient momentum to become visible in the chamber. On the other hand, the category of even events has different constituents. First, there is the direct process on the proton, leaving the neutron as an invisible spectator. Second, there is the direct process on the neutron where the proton spectator is visible. And third, there are primary reactions on either a proton or a neutron with a subsequent rescattering against the other nucleon. Spectator events may be individually identified when the slow proton is emitted into the backward hemisphere. The direct process on a target at rest only yields secondary protons in the forward hemisphere. (For the small contamination of direct protons, emitted backward by fermi motion, a correction is made.) From the events with a backward spectator, events with a forward spectator may be constructed.

In addition, a selection may be made of rescattering events by means of kinematical arguments. The quantity

$$
\epsilon=\sum_{f}\left(E_{f}-p_{f}^{3}\right)-m
$$

is evaluated, where the sum runs over all visible particles of the event. If all particles are visible and correctly identified, $\epsilon=0$, otherwise $\epsilon<0$. On the other hand, for rescattering events where the target is the deuteron rather than a single nucleon with mass $m$, $\epsilon$ may become larger than 0. A more detailed description of the experimental analysis of rescattering is found in Refs. 20 22].

\section{B. Direct process}

To compute the cross section for the direct process we use a convolution model [4, 5 ], in which the fermi motion and binding are taken into account by folding the semi-inclusive structure functions for a free nucleon with the momentum distribution of the nucleon in the 
nucleus. In this approach off-shell effects and possible final state interactions between the quarks in the struck nucleon and the spectator nucleons are neglected.

When using the fragmentation approach for the scattering off valence and sea quarks the cross section can be expressed as an integral over the light-cone momentum fraction carried by the nucleon (cf. Fig. 四a):

$$
\begin{aligned}
& \frac{d^{4} \sigma_{\mathrm{dir}}^{\nu d}(x, z)}{d x d z d^{2} \vec{p}_{\perp}}=\frac{G^{2} m E_{\nu}}{\pi} \frac{1}{1-x} \sum_{\tau=p, n} \int_{x+z}^{m_{d} / m} d y d^{2} \vec{k}_{\perp} f_{d}\left(y, \vec{k}_{\perp}\right) \\
& \left\{\frac{2}{3} \mathcal{F}_{2}^{\nu \tau}\left(\frac{x}{y}, \frac{z}{y-x},\left|\vec{p}_{\perp}-\frac{z}{y} \vec{k}_{\perp}\right|\right)+\frac{x}{3 y} \mathcal{F}_{3}^{\nu \tau}\left(\frac{x}{y}, \frac{z}{y-x},\left|\vec{p}_{\perp}-\frac{z}{y} \vec{k}_{\perp}\right|\right)\right\}
\end{aligned}
$$

where the structure functions are defined in Eqs. (2) and (3) and the generalized convolution function is given by 9 , 5

$$
f\left(y, \vec{p}_{\perp}\right)=m y \int \frac{d^{3} k}{\sqrt{k^{2}+m^{2}}} n(k) \delta\left(y-\frac{m_{d}-\sqrt{k^{2}+m^{2}}-k_{3}}{m}\right) \delta^{(2)}\left(\vec{k}_{\perp}-\vec{p}_{\perp}\right) .
$$

After integration over the transverse momentum one recovers the usual convolution function for the light-cone momentum [5]

$$
f_{d}(y)=2 \pi m^{2} y \int_{k_{\min }}^{\infty} \frac{k d k}{\sqrt{k^{2}+m^{2}}} n(k),
$$

where $k_{\min }=\left|\frac{\left(m y-m_{d}\right)^{2}-m^{2}}{2\left(m_{d}-m y\right)}\right|$. The factor $y$ in Eq. (15) which comes from the convolution of the hadronic tensor, is usually referred to as the flux factor. Alternatively one can convolute the semi-inclusive cross section; in this case the factor $y$ arises from the presence of the invariant mass of the neutrino plus nucleon system in the convolution integral (Ref. [6]).

In the present work the momentum density $n(k)$ is taken from Ref. [23] (Bonn potential). For the deuteron the convolution function $f_{d}(y)$ is sharply peaked at $y=1$, corresponding to a nucleon at rest, and falls of rapidly for $y \neq 1$ and as a result the cross section for the direct process differs very little from the one for the free nucleon case. The main difference is that, due to the fermi-motion, the calculated cross section extends to larger values of $x$. Furthermore, the number of protons produced in the backward direction with a momentum above the experimental cutoff of $150 \mathrm{MeV} / \mathrm{c}$ is negligible. 
As noted above it is possible to compare the results for the direct cross section on the neutron with the data set for odd events, to which the even events with a backward and a constructed forward spectator are added. The calculated $P(x, \Delta p)$ as a function of $x$ for the free neutron (shown in Fig. 3) and those for the bound neutron (shown in Fig. 5) differ slightly both in the value of the cut-off in $x$ (due to the fermi motion in the deuteron ) and in normalization (due to the different inclusive cross sections for $A=1$ and $A=2$ ). Again the smaller SIDIS cross section for the $\bar{\nu}$ case can be explained by the presence of the unfavoured fragmentation function.

\section{Spectator process}

In the absence of final state interactions the cross section for the spectator process is given by (cf. Fig. 四b)

$$
\begin{aligned}
\frac{d^{4} \sigma_{\text {spec }}^{\nu d}}{d x d z d^{2} \vec{p}_{\perp}}= & \frac{G^{2} m E_{\nu}}{\pi} \int_{x}^{m_{d} / m} d y\left\{\frac{2}{3} F_{2}^{\nu n}\left(\frac{x}{y}\right)+\frac{x}{3 y} F_{3}^{\nu n}\left(\frac{x}{y}\right)\right\} \\
& f_{d}\left(\frac{m_{d}}{m}-z, \vec{p}_{\perp}\right) \delta\left(z+y-m_{d} / m\right) .
\end{aligned}
$$

Hence the dependence of cross section on $z$ (when integrated over $p_{\perp}$ ) is determined by the light-cone momentum fraction of the neutron in the deuteron (approximately symmetric around $z=1$ ) and the corresponding shift in the arguments of the structure functions (which is asymmetric around $z=1$ ). One sees that the maximum l.c. momentum fraction of the spectator for given $x$ is given by $z_{\max }=m_{d} / m-x$ corresponding to a backward momentum 24] $p_{\max }=\frac{m^{2}-\left(m_{d}-x m\right)^{2}}{2\left(m_{d}-x m\right)}$. In particular, for $x=0$ one has $p_{\max }=\frac{3}{4} m$. Because the convolution function is strongly peaked at $y=1$ the spectator cross section decreases rapidly when going away from $z=1$.

In Fig. 6 the calculated ratio of the semi-inclusive and inclusive cross sections $P(x, \Delta p)$ for the spectator process is compared with the backward spectator spectrum as extracted from the even hadron set. $P(x, \Delta p)$ depends only weakly on $x$, since the scattering process on the neutron is essentially inclusive. The difference in $x$ dependence between neutrinos 
and anti-neutrinos can be attributed to the difference of the $x$ distributions between the $u$ and $d$ quarks in the proton and the neutron: spectator protons correspond to events where the $\nu(\bar{\nu})$ scatters off a $d(u)$ quark in the neutron whereas the inclusive deuteron cross section involves an average over proton and neutron events.

Also of interest is the ratio $R(z, \Delta x)$ obtained by integrating the semi-inclusive cross section over the transverse momentum and $x$, and dividing by the inclusive cross section

$$
R(z, \Delta x)=\int_{\Delta x} d x d p_{\perp} \sigma\left(x, z, p_{\perp}\right) / \int_{\Delta x} d x \sigma(x)
$$

Fig. 7 shows that $R(z, \Delta x)$ for the spectator process (only backwards), sharply peaked at $z=1$, is well reproduced. (The plateau around $z=1$ is due to the lower momentum cutoff at $150 \mathrm{MeV} / \mathrm{c}$.

Finally, in Fig. 8 we show the calculated ratio for the sum of direct and spectator cross sections. The peak at $z=1$ corresponds to detection of the spectator, whereas the ridge at small values of $x$ stems from the direct production of protons.

\section{Rescattering}

Above we have restricted ourselves to the PWIA, i.e. neglected effects from final state interactions between the spectator and the hadrons produced by the fragmentation. In the present context two aspects of fsi are of interest. First as shown in [22] the inclusive rescattering probability (which experimentally can be identified with the help of the variable

$\epsilon)$ is sensitive to the time (length) scale in the hadronization process. A second topic of interest is the modification of the spectator l.c. momentum distribution.

The deuteron is the simplest nuclear system where the hadronization process in a medium can be studied, and the nuclear structure can be treated without approximation. Below we discuss the consequences of final state interactions (rescattering) for both the spectator spectrum and the rescattering probability. 


\section{Inclusive rescattering probability}

The simplest approach to rescattering is to assume that the hit nucleon hadronizes instantaneously into a number of color singlets which can rescatter incoherently on the spectator. It was shown in [22] that in this independent rescattering model the observed rescattering fraction $P_{\text {resc }}$ (the number of rescatter events over the total number of events) is grossly overestimated. In the eikonal approximation the incoherent rescattering probability is [25]:

$$
P_{\text {resc }}\left(x, Q^{2}\right)=\frac{N\left(x, Q^{2}\right) \sigma}{4 \pi}\left\langle\frac{1}{r^{2}}\right\rangle_{d}
$$

where $\sigma$ is the fragment-spectator cross section and $N\left(x, Q^{2}\right)$ is the multiplicity. The latter can be parametrized as 26,27

$$
N\left(x, Q^{2}\right)=1.1+1.35 \ln W^{2}\left(x, Q^{2}\right)
$$

where $W\left(x, Q^{2}\right)$ is the invariant mass of the hadronic system:

$$
W\left(x, Q^{2}\right)=\sqrt{m_{N}^{2}+Q^{2}(1 / x-1)} .
$$

This would predict too large a magnitude of $P_{\text {resc }}$ and moreover, a logarithmic increase in the rescattering probability as a function of the energy transfer $\nu \sim Q^{2} / x$. In contrast, the experiment 22] shows a saturation for large $\nu$ and a very small $x$ dependence (cf. Fig. 9).

An alternative to the incoherent rescattering model is to replace $N\left(x, Q^{2}\right)$ by $N_{\text {eff }}=1$, independent of $x$. Using for the fragment-nucleon cross section $\sigma$ a value of $45 \mathrm{mb}$ one obtains $P_{\text {resc }} \approx 0.12$. This agrees with the average experimental value. This suggests that effectively only one of the produced colorless fragments will interact. Alternatively, this can be viewed as the rescattering of an object (the exited nucleon or string) with the transverse size of the hit nucleon moving through the nucleus.

As shown in 22 the most plausible explanation of the small rescattering probability is the finite formation time for the newly created particles. We have estimated this effect by means of a Monte Carlo calculation and use the VENUS hadronization code (which is based on string fragmentation) to generate a space-time spectrum of fragments. To simulate 
coherence we assume that only the fragment which is formed closest to the scattering centre will rescatter. Introducing the distribution $F_{\mathrm{x}}\left(x, r_{0}\right)$ which gives the probability that the first fragment is of type $\mathrm{x}$ and is formed at a distance $r_{0}$ from the scattering center, the rescattering probability can be expressed as [25]:

$$
P_{\text {resc }}(x)=\int_{0}^{\infty} d r_{0} F_{\mathrm{x}}\left(x, r_{0}\right) \int_{r_{0}}^{\infty} d r \frac{\sigma \rho_{d}(r)}{4 \pi r^{2}}
$$

In Fig. 9 the inclusive rescattering probability as given by Eq. (22) is plotted as a function of $x$ (where we have folded in the experimental $Q^{2}$ distribution). Also shown is the rescattering probability for the independent rescattering model with $N=1$. One sees that this simple model for rescattering does not explain the data: the rescattering probability is underestimated and the $x$ dependence is not present in the data.

This strongly indicates that the present approach is too simple: we have neglected all interactions of the string (excited nucleon) before hadronization and assumed that only one fragment will rescatter. Any interaction of the string will however, increase the rescattering probability and smoothen the $x$-dependence.

\section{Spectator modification}

The spectator process has been proposed as a means to measure correlations in nuclei. In the independent pair approximation where two nucleons move back to back in the nucleus in their cm system after striking one nucleon its partner can leave the nucleus with its original lc momentum if fsi are neglected. In the backward direction one thus measures the nucleon lc momentum distribution. There are several ways to study whether this simple picture is

affected by fsi. A simple criterium is the ratio of $x$ values for events with and without a slow backward proton, $v_{z}=\langle x\rangle_{\mathrm{bp}} /\langle x\rangle$, which in the case of spectator events is proportional to $2-z$. In case of ${ }^{20} \mathrm{Ne}$ this relation has been confirmed qualitatively for events containing only one slow proton 28.

The most obvious contribution to the rescattering process, that gives rise to slow protons, is that of the elastic rescattering of fragments on the spectator. The resulting events are 
thus removed from the spectator and added to the rescattering sample. The spectrum of rescattered spectators can be calculated using a generalization of $F_{\mathrm{x}}\left(x, r_{0}\right)$ which includes the distribution of the longitudinal momentum $p_{3}$ carried by the fragments 229.

Using this distribution we can write the modified backward proton spectrum as as a sum of rescattered and undistorted spectators

$$
\sigma^{\mathrm{bp}}=\sigma^{\mathrm{resc}}+\tilde{\sigma}^{\mathrm{spec}}
$$

where $\tilde{\sigma}^{\text {spec }}=\left(1-P_{\text {resc }}\right) \sigma^{\text {spec }}$ is the cross section for spectators, that are unaffected by rescattering, and $\sigma^{\text {resc }}$ the spectrum of protons that arises from the rescattering process.

As a first estimate of the effect of rescattering we calculated the distorted spectator distribution (including only elastic rescattering). For the cross section we used the parametrization from Ref. [30] for all types of fragments (including resonances and mesons). In Fig. 10 we plot the spectrum of backward protons

$$
R(z, \Delta x)=\left(\int d x \frac{d \sigma^{\mathrm{bp}}}{d x d z}\right)\left(\int d x \frac{d \sigma^{\mathrm{deut}}}{d x}\right)^{-1}
$$

as a function of $z$. It is seen that, as one expects, the spectrum is shifted towards the forward hemisphere $(z<1)$. However, because of the rather small effect, and the number of approximations in the calculation it is not possible to make a quantitative comparison with the (anti-)neutrino data.

\section{CONCLUDING REMARKS}

Motivated by a reanalysis of experimental data we have investigated the absolute cross section for semi-inclusive deep inelastic charged current (anti) neutrino scattering on hydrogen and deuterium with the observation of a slow proton in the final state, as a function of the Bjorken $x$ and the light-cone momentum fraction $z$.

On a free nucleon, we assume that the cross section can be factorized into a quark momentum distribution and a fragmentation function for the spectator quarks. At small 
values of $x$ the cross section is dominated by sea-quark effects; the latter can be simulated by a pion cloud model, and by a four-quark fragmentation function. The strong bias of the ratio $P(x, z)$ of the coincidence cross section and the inclusive cross section for small $x$ values has basically a kinematic origin.

The deuteron target offers several new interesting effects. First, the data analysis makes it possible to isolate deep inelastic scattering on the neutron with subsequent fragmentation into a proton. Second, there is an additional source of slow protons in the final state, namely the emitted spectator nucleon. In the backward hemisphere where this process dominates, the cross section is directly proportional to the light-cone momentum distribution of the nucleons. In most cases qualitative agreement with the data is obtained; a more quantitative comparison can only be made using higher accuracy data. Finally, an analysis of rescattering events indicates that the naive incoherent rescattering of produced hadrons is not consistent with experiment.

There are several reasons why it is interesting to extend the present study to heavier nuclei. In the plane wave impulse approximation the spectator momentum distribution depends sensitively on details of the nuclear spectral function $P(k, E)$. In addition one expects to obtain more information about final state interactions between spectator nucleons and produced hadrons, in which the formation time plays a crucial role.

\section{ACKNOWLEDGMENTS}

The authors thank the Amsterdam-Bergen-Bologna-Padova-Pisa-Saclay-Torino Collaboration for supplying the experimental data. They thank the staff of the CERN SPS and the bubble chamber BEBC for establishing the experiment. The authors wish to thank O. Scholten, C. Korpa, M. Strikman, L. Frankfurt, P.J. Mulders and J. Guy for stimulating discussions. This work was supported by the Foundation for Fundamental Research (FOM) and the Netherlands Organization for the Advancement of Scientific Research (NWO). 


\section{REFERENCES}

[1] T. Sloan, G. Smadja and R. Voss, Phys. Rep. 162, 45 (1988).

[2] R.P. Bickerstaff and A.W. Thomas, J. Phys. G15, 1523 (1989).

[3] A.E.L. Dieperink and A.G. Miller, Phys. Rev. C 44, 866 (1991).

[4] G.D. Bosveld, A.E.L. Dieperink and O. Scholten, Phys. Lett. B264, 11 (1991).

[5] G.D. Bosveld, A.E.L. Dieperink and O. Scholten, Phys. Rev. C 45, 2616 (1992).

[6] L.L. Frankfurt and M.I. Strikman, Phys. Rep. 76, 215 (1981).

[7] L. Heller and A.W. Thomas, Phys. Rev. C 41, 2756 (1990).

[8] L. Frankfurt, G.A. Miller and M. Strikman, Phys. Rev. Lett. 68, 17 (1992).

[9] U. Oelfke, P.U. Sauer and F. Coester, Nucl. Phys. A518, 593 (1990).

[10] J. Levelt and P.J. Mulders, preprint NIKHEF 92-P9, revised version.

[11] L. Frankfurt and M. Strikman, Phys. Rep. 160, 235 (1988).

[12] A. Bartl, H. Fraas and W. Majerotto, Phys. Rev. D 26, 1061 (1982).

[13] W. Melnitchouk, A.W. Thomas and N.N. Nikolaev, Z. Phys. A 342, 215 (1992).

[14] R. Blankenbecler and S.J. Brodsky, Phys. Rev. D 10, 2973 (1974).

[15] S.J. Brodsky and J.F. Gunion, Phys. Rev. D 17, 848 (1978).

[16] C. Ishii, K. Saito and F. Takagi, Phys. Lett. B216, 409 (1989).

[17] C. Korpa, A.E.L. Dieperink and O. Scholten, Z. Phys. A 343, 461 (1992).

[18] J. Guy et. al., Phys. Lett. B229, 421 (1989).

[19] D. Allasia et al., Nucl. Phys. B268, 1 (1986), and references therein.

[20] A.G Tenner, NIKHEF-H/86-7 (unpublished). 
[21] A.G Tenner, NIKHEF-H/88-6 (unpublished).

[22] A.G. Tenner and N.N. Nikolaev, Nuovo Cim. A 105, 1001 (1992).

[23] R. Machleidt, K. Holinde and Ch. Elster, Phys. Rep. 149, 1 (1987).

[24] C.E. Carlson, K.E. Lassila and U.P. Sukhatme, Phys. Lett. B263, 277 (1991).

[25] O. Benhar, A. Fabrocini, S. Fantoni, G.A. Miller, V.R. Pandharipande and I. Sick, Phys. Rev. Lett. 69, 881 (1992).

[26] J. Bell et al., Phys. Rev. D 19, 1 (1979).

[27] J.W. Chapman et al., Phys. Rev. Lett. 36, 124 (1976).

[28] E. Matsinos et al., Z. Phys. C 44, 79 (1989).

[29] G.D. Bosveld and A.E.L. Dieperink (in preparation).

[30] P. Závada, Z. Phys. C 32, 135 (1986). 


\section{FIGURES}

FIG. 1. Debris fragmentation in DIS

FIG. 2. Ratio $P(x, \Delta p)$ of the semi-inclusive production cross section of slow protons $(\nu(\bar{\nu})+p \rightarrow \mu+p+X)$ off free protons and the corresponding total inclusive cross section for two momentum bins of the slow proton (left 150-350 MeV/c, right 350-600 MeV/c). For the calculation the branching ratios from Tables $[\mathrm{I}$ (set 1) and [II (set 2) have been used. Comparison is made

with the data from Ref. [18] which have been normalized to the the calculated ratio for the low bin in neutrino scattering.

FIG. 3. Ratio $P(x, \Delta p)$ for slow proton production off the neutron, equivalent to Fig. 2. A comparison is made with experimental deuterium data as explained in Sec. 3. No normalization has been performed, the theoretical curves act as absolute predicions of the data.

FIG. 4. Direct (a) and spectator process (b) for semi-inclusive deep-inelastic scattering on $A=2$.

FIG. 5. The calculated ratio $P(x, \Delta p)$ for the direct process on the neutron bound in the deuteron compared to the data.

FIG. 6. The ratio $P(x, \Delta p)$ for the spectator process in the deuteron compared to the data (backward spectators only).

FIG. 7. The ratio $R(z, \Delta x)$ for the spectator process as a function of $z$ in two different $x$ bins compared to the data.

FIG. 8. Calculated ratio $R(x, z)=\sigma(x, z) / \sigma(x)$ of sum of the cross sections for the spectator and direct process in $\nu+d \rightarrow \mu^{-}+p+X$ and the inclusive cross section $\nu+d \rightarrow \mu^{-}+X$, with a lower momentum cutoff of $150 \mathrm{MeV} / \mathrm{c}$ for the observed proton. 
FIG. 9. Rescattering probabilities as a function of $x$, calculated using the independent rescattering model with $N_{\text {eff }}=1$ (dashed lines) and the formation time model (solid lines), compared to the data.

FIG. 10. The ratio $R(z, \Delta x)(0<x<0.1)$ for backward protons calculated without (solid line) and with rescattering (dashed line). 


\section{TABLES}

TABLE I. Relative weights $\gamma_{\{q q\}}^{\mathrm{x}}$ for diquark fragmentation functions $D_{\{q q\}}^{\mathrm{x}}$.

\begin{tabular}{ccccccc}
\hline \hline$\{q q\}$ & $\Delta^{++}$ & $\Delta^{+}$ & $\Delta^{0}$ & $\Delta^{-}$ & $p$ & $n$ \\
\hline$u u$ & $\frac{18}{27}$ & $\frac{6}{27}$ & 0 & 0 & $\frac{3}{27}$ & 0 \\
$u d$ & 0 & $\frac{2}{18}$ & $\frac{2}{18}$ & 0 & $\frac{7}{18}$ & $\frac{7}{18}$ \\
$d d$ & 0 & 0 & $\frac{6}{27}$ & $\frac{18}{27}$ & 0 & $\frac{3}{27}$ \\
\hline \hline
\end{tabular}

TABLE II. Relative weights $\gamma_{\{4 q\}}^{\mathrm{x}}$ for naive four-quark fragmentation

\begin{tabular}{cccccccc}
\hline \hline process & $\{4 q\}$ & $\Delta^{++}$ & $\Delta^{+}$ & $\Delta^{0}$ & $\Delta^{-}$ & $p$ & $n$ \\
\hline$\nu p$ & $p / d_{s}, p / \bar{u}_{s}$ & 0 & $1 / 2$ & 0 & 0 & $1 / 2$ & 0 \\
$\bar{\nu} p$ & $p / u_{s}, p / \bar{d}_{s}$ & 0 & $1 / 2$ & 0 & 0 & $1 / 2$ & 0 \\
$\nu n$ & $n / d_{s}, n / \bar{u}_{s}$ & 0 & 0 & $1 / 2$ & 0 & 0 & $1 / 2$ \\
$\bar{\nu} n$ & $n / u_{s}, n / \bar{d}_{s}$ & 0 & 0 & $1 / 2$ & 0 & 0 & $1 / 2$ \\
\hline \hline
\end{tabular}

The notation $\left\{p / d_{s}\right\}$ denotes the debris after hitting a $d_{s}$ in the proton.

TABLE III. Relative weights $\gamma_{\{4 q\}}^{\mathrm{x}}$ using isospin branchings of the pion cloud model.

\begin{tabular}{cccccccc}
\hline \hline process & $\{4 q\}$ & $\Delta^{++}$ & $\Delta^{+}$ & $\Delta^{0}$ & $\Delta^{-}$ & $p$ & $n$ \\
\hline$\nu p$ & $p / d_{s}, p / \bar{u}_{s}$ & $\frac{3 \kappa}{4 \kappa+1}$ & $\frac{\kappa}{4 \kappa+1}$ & 0 & 0 & $\frac{1}{4 \kappa+1}$ & 0 \\
$\bar{\nu} p$ & $p / u_{s}, p / \bar{d}_{s}$ & 0 & $\frac{\kappa}{2 \kappa+5}$ & $\frac{\kappa}{2 \kappa+5}$ & 0 & $\frac{1}{2 \kappa+5}$ & $\frac{4}{2 \kappa+5}$ \\
$\nu n$ & $n / d_{s}, n / \bar{u}_{s}$ & 0 & $\frac{\kappa}{2 \kappa+5}$ & $\frac{\kappa}{2 \kappa+5}$ & 0 & $\frac{4}{2 \kappa+5}$ & $\frac{1}{2 \kappa+5}$ \\
$\bar{\nu} n$ & $n / u_{s}, n / \bar{d}_{s}$ & 0 & 0 & $\frac{\kappa}{4 \kappa+1}$ & $\frac{3 \kappa}{4 \kappa+1}$ & 0 & $\frac{1}{4 \kappa+1}$ \\
\hline \hline
\end{tabular}


This figure "fig1-1.png" is available in "png" format from: http://arxiv.org/ps/nucl-th/9311030v1 
This figure "fig2-1.png" is available in "png" format from: http://arxiv.org/ps/nucl-th/9311030v1 
This figure "fig1-2.png" is available in "png" format from: http://arxiv.org/ps/nucl-th/9311030v1 
This figure "fig2-2.png" is available in "png" format from: http://arxiv.org/ps/nucl-th/9311030v1 
This figure "fig1-3.png" is available in "png" format from: http://arxiv.org/ps/nucl-th/9311030v1 
This figure "fig2-3.png" is available in "png" format from: http://arxiv.org/ps/nucl-th/9311030v1 
This figure "fig1-4.png" is available in "png" format from: http://arxiv.org/ps/nucl-th/9311030v1 